\title{
In vivo fiber tractography of the right and left ventricles using diffusion tensor MRI of the entire human heart
}

\author{
Choukri Mekkaoui ${ }^{1 *}$, Timothy G Reese ${ }^{1}$, Marcel P Jackowski ${ }^{2}$, Himanshu Bhat ${ }^{3}$, William J Kostis ${ }^{1}$, David E Sosnovik \\ From 17th Annual SCMR Scientific Sessions \\ New Orleans, LA, USA. 16-19 January 2014
}

\section{Background}

Diffusion Tensor MRI (DTI) tractography of the human heart in vivo has previously been performed with either large slice gaps or limited coverage [1,2]. The aim of this study was to investigate the feasibility of performing DTI of the entire human heart in vivo without slice gaps. This, we hypothesized, would enhance the characterization of fiber architecture in the left ventricle (LV), allow myofiber organization in the right ventricle (RV) to be characterized in vivo, and further elucidate microstructural differences in the heart between systole and diastole.

\section{Methods}

DTI was performed on a clinical 3T scanner (Skyra, Siemens) using a fat-suppressed, zone-selected, diffusionencoded stimulated echo sequence with 10 diffusion encoding directions, TE/TR 33/80 ms, GRAPPA rate 2, b-value $500 \mathrm{~s} / \mathrm{mm}^{2}$, resolution $2.8 \times 2.8 \times 8 \mathrm{~mm}^{3}, 8$ averages and multiple breath-holds. The entire LV and RV were covered in 13 contiguous short-axis slices. Images were acquired in the systolic and diastolic sweet spots [3] of the cardiac cycle and were spatiotemporally coregistered [4]. Tractography was performed by numerically integrating the primary eigenvector field into streamlines using an adaptive $5^{\text {th }}$ order RungeKutta method. The impact of cumulative image averages (1-8) on the reliability of the fractional anisotropy (FA) and fiber helix angle (HA) indices was assessed.

\section{Results}

A composite view of the anterior thorax and heart is shown in Figure 1A. The contrast between the helical pattern of the fibers in the heart (color-coded by HA) and the linear organization of skeletal muscle fibers in the chest wall can be seen. The RV consisted of a bilayer of obliquely-oriented fibers, lacking circumferential fibers (Figure 1B). In contrast, fibers in the midwall of the LV were circumferential, forming a distinct band between the subendocardial and subepicardial fibers (Figure 1C). Figure 1D shows the tractogram of the entire heart, depicting the intertwined arrangement of myofibers at the anteroseptal RV-LV junction. Figure $2 \mathrm{~A}$ and $2 \mathrm{~B}$ show that FA values in both sweet spots were significantly overestimated when the number of averages used was $<5(\mathrm{p}<0.01)$. In contrast, convergence to a stable FA value ( $\mathrm{LV}$, diastole: $0.42 \pm 0.03$, systole: $0.41 \pm 0.03$; RV, diastole: $0.32 \pm 0.11$, systole: $0.36 \pm 0.02$ ) was observed from 5-8 averages. The transmural gradient in HA exhibited a similar pattern of convergence but required more averages (Figure $2 \mathrm{C}, \mathrm{D}$ ).

\section{Conclusions}

DTI-tractography of the entire human heart can be performed in vivo, without slice gaps, and allows both the $\mathrm{LV}$ and the RV to be characterized. A minimum of 5 averages at each slice location is required for accurate quantification. The ability to characterize fiber architecture in the LV and RV in vivo has the potential to provide new insights into a range of diseases affecting both the pulmonary venous and arterial circulations.

\section{Funding}

R01 HL093038 (D.E.S.), R01HL112831 (D.E.S.), P41RR14075 (Martinos Center). 


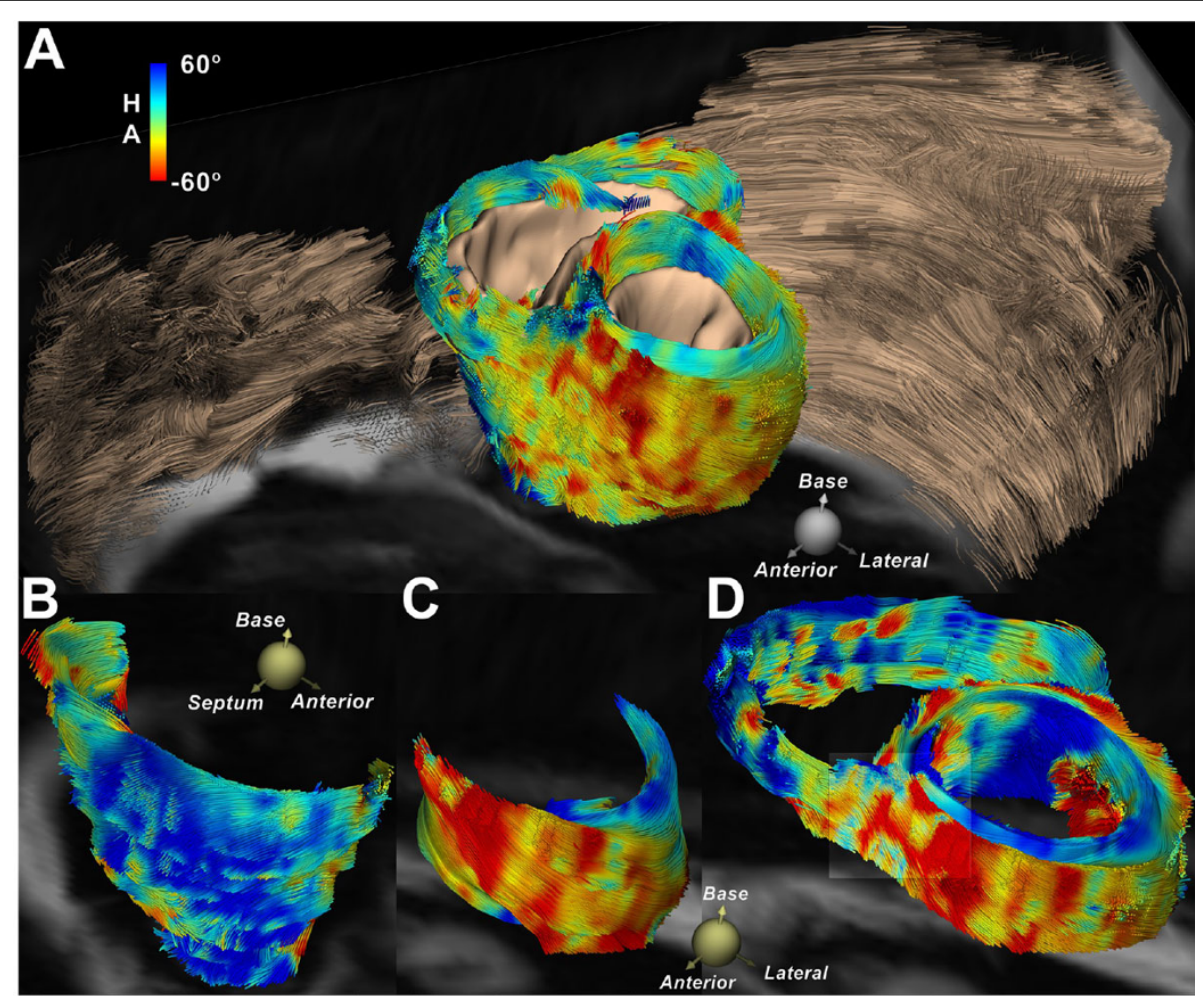

Figure 1 (A) A composite view of the anterior thorax and heart shows the contrast between the helical pattern of the fibers in the myocardium (color-coded by HA) and the linear organization of skeletal muscle fibers in the chest wall. (B) The fiber architecture of the RV can be well resolved in vivo and lacks the circumferential fibers seen in the LV (C). (D) Tractography within a slab located at the basal-level depicting the transmural myofiber arrangement of both RV and LV. The intertwined arrangement of myofibers at the anteroseptal RV-LV junction is slightly magnified.

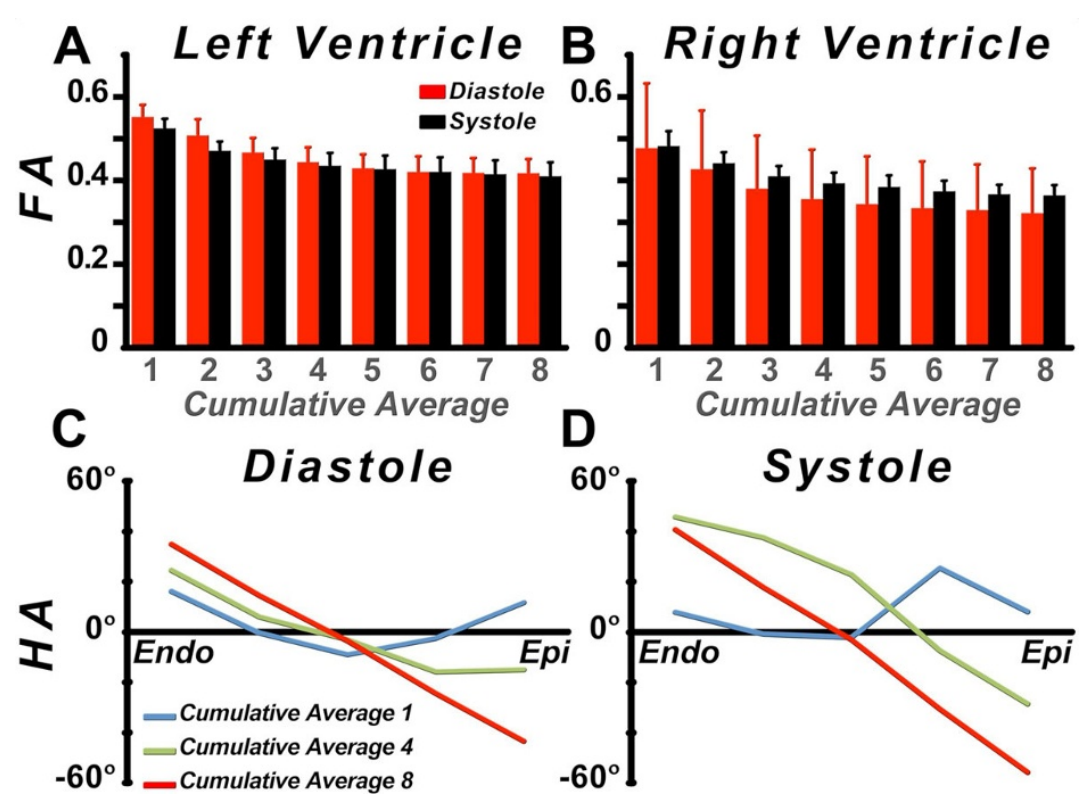

Figure 2 (A, B) Cumulative averages (1-8) of FA for the LV and the RV, respectively. (A) In the LV, FA values stabilize after 5 averages in diastole as well as in systole. (B) In the RV, FA also stabilizes after 5 averages, albeit with a higher standard deviation in diastole, but FA values are $9 \%$ lower in diastole than in systole. (C, D) Transmural distribution of HA for 1, 4, and 8 cumulative averages in diastole and systole, respectively. For optimal estimation of $\mathrm{HA}, 8$ averages are required since the $\mathrm{HA}$ is a function of the primary eigenvector, and more degrees of freedom need to be estimated. 


\section{Authors' details}

${ }^{1}$ Radiology, Harvard Medical School - Massachussets General Hospital -

Martinos Center for Biomedical Imaging, Charlestown, Massachusetts, USA.

${ }^{2}$ Department of Computer Science, University of São Paulo, São Paulo, São

Paulo, Brazil. ${ }^{3}$ Siemens Medical Solutions USA Inc., Charlestown,

Massachusetts, USA.

Published: 16 January 2014

\section{References}

1. Toussaint, et al: MEDIA 2013.

2. Nielles-Vallespin, et al: MRM 2012

3. Tseng, et al: MRM 1999.

4. Mekkaoui, et al: JCMR 2012.

doi:10.1186/1532-429X-16-S1-P17

Cite this article as: Mekkaoui et al:: In vivo fiber tractography of the

right and left ventricles using diffusion tensor MRI of the entire human

heart. Journal of Cardiovascular Magnetic Resonance 2014 16(Suppl 1):P17.

\section{Submit your next manuscript to BioMed Central} and take full advantage of:

- Convenient online submission

- Thorough peer review

- No space constraints or color figure charges

- Immediate publication on acceptance

- Inclusion in PubMed, CAS, Scopus and Google Scholar

- Research which is freely available for redistribution

Submit your manuscript at www.biomedcentral.com/submit 